\title{
TURBULENT WIND FIELD SIMULATION OF WIND TURBINE STRUCTURES WITH CONSIDERATION OF THE EFFECT OF ROTATING BLADES
}

\author{
Tao Huo ${ }^{1,2,3}$, Le-Wei Tong ${ }^{1,2 *}$ and Fidelis R. Mashiri ${ }^{4}$ \\ ${ }^{I}$ State Key Laboratory of Disaster Reduction in Civil Engineering, Tongji University, Shanghai 200092, China \\ ${ }^{2}$ College of Civil Engineering, Tongji University, Shanghai 200092, China \\ ${ }^{3}$ East China Architectural Design \& Research Institute Co., Ltd, Shanghai 200011, China \\ ${ }^{4}$ School of Computing, Engineering and Mathematics, Western Sydney University, Sydney, NSW 2751, Australia \\ *(Corresponding author: E-mail: tonglw@tongji.edu.cn)
}

\section{A B S T R A C T}

In order to achieve the wind-induced vibration response analysis and fatigue analysis, this study conducts the wind field simulations around tubular tower and rotating blades of typical pitch-controlled 1.25MW wind turbine structures, respectively. Based on field test data, there is a large difference between the turbulent wind spectrum for the rotating blades and classic wind spectrum adopted by the non-rotating blades and tubular tower. In this study, first, the auto and crossrotational Fourier spectrums are deduced based on the physical mechanism, with particular focus on the influences of the rotational effect and the correlation between different points located on the same and different blades. Then, the Davenport type coherence function is optimized. The high accuracy of the rotational Fourier spectrum model is verified by comparing with the real data. Relevant parameter analysis of the rotational Fourier spectrum is conducted. Finally, turbulent wind fields around the tubular tower based on the Kaimal spectrum and the rotating blades based on the rotational Fourier spectrum are simulated by means of the harmony superposition method. The results indicate that the calculated wind spectrums have good agreement with the target wind spectrums. Therefore, the proposed approach in this study is feasible for the turbulent wind field simulation of wind turbine structures.
A R T I C LE H I S T O RY

$\begin{array}{ll}\text { Received: } & \text { 31 January } 2018 \\ \text { Revised: } & \text { 12 July } 2018 \\ \text { Accepted: } & \text { 25 July } 2018\end{array}$

\section{K E Y W O R D S}

Wind turbine structures;

Turbulent wind field simulation;

Rotational Fourier spectrum;

Coherence function;

optimization;

Parameter analysis:

Harmony superposition method

\section{Introduction}

As more and more public concern and awareness about energy and environmental issues has increased, wind power, which is a renewable source of energy, has gained prominence and has been extensively developed [1,2]. In recent years, large-scale wind turbine structures have been widely used around the world, with the characteristics of large moment of inertia, broad range of working wind speed and high-rise towers [3,4]. It is worth noting that cyclic loadings, which are exerted on the rotating blades, and fluctuating wind load can inevitably bring about fatigue failure of the wind turbine tubular tower [5]. In general, the fatigue issue of tubular towers is not only related to the fatigue strength, but also dependent on the load effect (see Figure 1). While continuous progress has been made looking into the fatigue strength of the various materials, relevant investigations on the load effect induced by the cyclic loading of rotating blades and fluctuating wind load are generally insufficient. Therefore, in order to guarantee the fatigue reliability of the wind turbine tubular towers, it is essential to analyze wind-induced dynamic responses. To achieve this purpose, wind field simulations around the wind turbine structure are necessary, especially for the fluctuating component.

It is worth mentioning that due to the rotational effect of blades, the turbulent wind field model of wind turbine structures is quite different from that of the ordinary wind-sensitive structures such as high-rise buildings and bridges [6]. Field tests confirmed that compared with the turbulent wind spectrum of the non-rotating blades, energy distribution of turbulent wind from rotating blades produces fundamental change, especially in high frequency components [7]. Moreover, wind-induced fatigue loads obtained through considering rotational effect of blades are obviously greater than the corresponding values caused by the non-rotating state, which could lead to unsafe fatigue life prediction [8]. Therefore, rotational effect of blades should be considered to accurately obtain the aerodynamic loads and carry out the wind-induced fatigue analysis of wind turbine structures. However, many researchers still adopt the classical turbulent wind spectrum models [3,9], such as the Von Karman and the Kaimal wind spectrums, to simulate the wind field around the blades, and relevant studies on rotational spectrum models of blades are still insufficient. Therefore, it is necessary to take an in-depth look into the theoretical rotational sampled wind spectrum to consider the impact of the blade rotational effect on turbulent wind field of wind turbine structures.
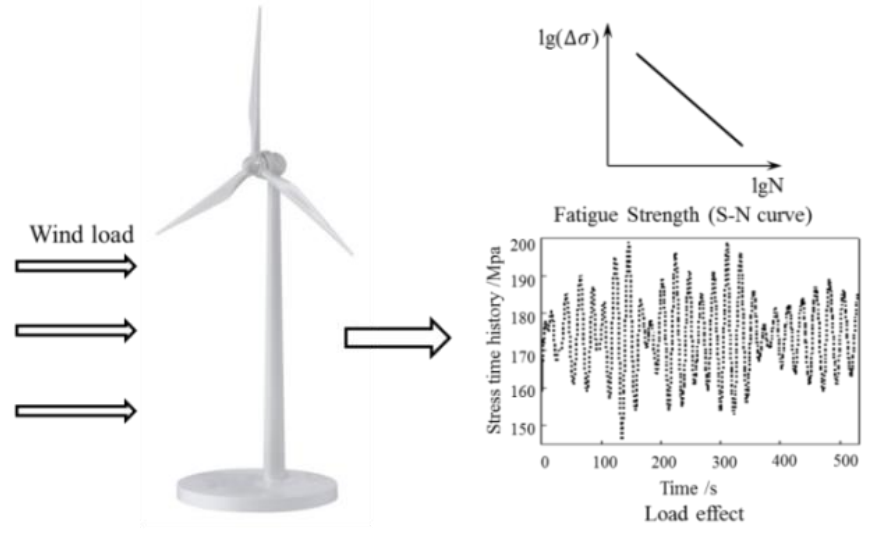

Fig.1 Fatigue calculation diagram

At present, there are two fundamental rotationally sampled wind spectrum models, namely, the Pacific Northwest Laboratory $(P N L)$ model $[5,8,10-13]$ and the Sandia National Laboratory (SNL) model $[14,15]$. With regard to the PNL model, Connell [7] found that there are significant differences between turbulent wind spectrum for the rotating blades and wind spectrum for the non-rotating blades, and that the energy redistribution is obvious. In order to describe the wind speed of rotating blades at a theoretic level, Connell [10] established a mathematical model of the rotational sampled wind spectrum in a rotating coordinate system. Powell [11] compared the theoretical model results to the measured data to validate the correctness of PNL model. Parameter analysis results show that turbulent wind speed variance and turbulent integral scales in longitudinal and lateral directions are important input parameters. Based on the PNL model, Powell and Connell [12] simulated the wind field of wind turbine structures and obtained the fluctuating wind speed taking the rotating effect of blades into account. However, only auto-spectrum was applied in the wind field simulation without considering correlation between the different fluctuating wind speeds. Based on the research findings proposed by Connell [10], Burton et al. [16] deduced the rotational cross-spectrum model that considered the correlation of different points located in the same blade. However, the correlation between the different points located on the different blades was not considered in the model. It is worth mentioning that the rotationally sampled spectrum of PNL model, which is an analytical formula, is generated through Fourier transformation of an autocorrelation function taken from Von Karman 
spectrum (one type of origin spectrum). For origin spectrum, this requirement, which influences further popularization and application, is extremely strict.

Veers [14] adopted a rotational sampling method to develop another theoretically rotational spectrum model, namely, the SNL model. Phase lag terms were introduced to consider the rotational effect of blades. Veers [15] presented that through improving two kinds of variables, the differences between calculated results and measured results can be controlled because of open origin spectrum and coherence function. Kelley [17] successfully extended the velocity components of the SNL model from the longitudinal direction to three dimensions. However, the SNL model has the limitations of timeconsuming, dissatisfying with Navier-Stokes equation, and artificially defining the coherence function $[14,15]$.

$\mathrm{He}$ [18] put forward a rotational Fourier spectrum of wind turbine structures based on the physical mechanism, however, the derivation process was relatively complex and no comparison with the measured results was conducted.

Therefore, it is essential to establish a theoretically rotational wind spectrum model in this study, which has the advantages of a simple derivation process, clear physical mechanism, open origin function and reliable simulated results, to simulate the wind field of wind turbine structures.

In order to conduct the wind-induced response analysis in time domain, numerical simulation algorithm of wind field based on Monte-Carlo theory should be developed. At present, the main methods adopted in the fluctuating wind speed simulation are Linear Filter Method [19], Harmony Superposition Method [20], and Wavelet Analysis Method [21]. Based on the linear filtering technique, Linear Filter Method uses Auto-Regressive algorithm $(A R)$, Moving Average algorithm $(M A)$ and Auto-Regressive Moving Average algorithm (ARMA) to describe the stationary random process. Wavelet Analysis Method adopts good localization characteristics of the wavelet and inverse wavelet transform to simulate random processes. Compared with other methods, Harmony Superposition Method, which is widely used in design standards and professional software of wind turbine structures, has its merits of high accuracy, simple algorithm, and strict mathematical deduction. Veers [14] firstly applied this method to the wind turbine structure.

In light of the above, based on the theoretical rotational spectrum proposed by He [18], a simpler and more accurate rotational Fourier spectrum model optimized by the current authors is established to consider the influence of blade rotating effect and the correlation between different points located on the same and different rotating blades in this study. Based on the optimized coherence function, a comparison between the rotational Fourier spectrum model and real data is conducted to confirm high accuracy of the rotational Fourier spectrum model. Moreover, parameter analyses are carried out in details. Finally, turbulent wind fields around the tubular tower using the Kaimal spectrum and the rotating blades adopting rotational Fourier spectrum are simulated based on the Harmony Superposition Method.

\section{Rotational Fourier spectrum model}

\subsection{Physical mechanism}

With blades rotating, the spatial position of any point located on the blade varies. Hence, the wind speed of any point on the blade rotational plane not only reflects the fluctuating property of wind speed itself but also involves the wind speed fluctuation induced by periodic variation of a spatial point coordinate. To accurately describe the wind speed time series of rotating blades, the Cartesian coordinate system should be transformed into the rotating coordinate system first, and then the sampling points are regularly selected on the rotating plane of the rotor, as shown in Figure. 2. When the blade rotates to the sampling point, the wind speed of sampling point at this time should be extracted. According to the time sequence, a set of wind speed time series are obtained. Finally, the rotational Fourier spectrum is generated through Fourier transformation of the new wind speed time series. In fact, the greatest advantage of rotational Fourier spectrum is that the kinematic problem of rotating blades can be transformed into a static problem.

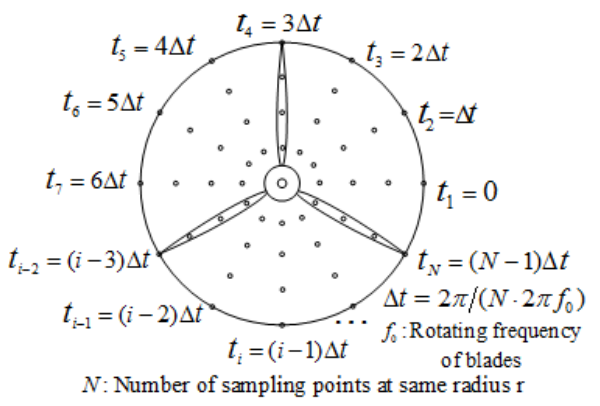

Fig.2 Sampling points position in rotating plane

\subsection{Rotational Fourier auto-spectrum}

In general, it is assumed that the wind field $U(x, y, z, t)$ is uniformly incompressible and isotropic. The mean wind speed is represented as $\bar{U}(z)$, and the fluctuating wind speed, which is represented as $u(x, y, z, t)$, is usually assumed to be a Gauss random process with zero mean value. In addition, for fluctuating wind field around blades, only the longitudinal component has obvious rotational effect, and lateral and vertical components are relatively complex. Herein, it is defined that the blades rotational plane is perpendicular to longitudinal direction, and the lateral direction is parallel to the blades rotational plane. Therefore, only the longitudinal fluctuating wind speed of the blades conducts the rotational Fourier spectrum analysis.

It is assumed that the blades rotate at a constant frequency, $f_{0}$. At any radius, $\mathrm{r}, u_{1}$ is the wind speed time series at time, $\mathrm{t}$, and $u_{2}$ is the wind speed time series at time, $t+\tau$ and radius, r. $\tau$ is the time interval. The cross-correlation function between $u_{1}$ and $u_{2}$ is represented as follows:

$R_{u_{1} u_{2}}=E\left[u_{1}(t) u_{2}(t+\tau)\right]$

According to the Wiener-Khintchine formula [22], the cross-power spectrum density between $u_{1}$ and $\boldsymbol{u}_{2}$ is as follows:

$S_{u_{1} u_{2}}(f)=\int_{-\infty}^{+\infty} R_{u_{1} u_{2}}(\tau) \cdot e^{-i 2 \pi f \tau} d \tau$

The corresponding inverse Fourier transformation is as follows:

$R_{u_{1} u_{2}}(\tau)=\int_{-\infty}^{+\infty} S_{u_{1} u_{2}}(f) \cdot e^{i 2 \pi f \tau} d f$

It is assumed that the fluctuating wind velocity obtained by sampling in the blade rotational plane is isotropic. Therefore, auto-power spectral density is independent of the spatial position of sampling points. The classical fluctuating wind spectrum density, such as the Von Karman wind spectrum [23] and the Kaimal wind spectrum [24, 25], is adopted as auto-power spectrum in this study. The relational expression between the fluctuating wind velocity cross-power spectrum and auto-power spectrum (origin spectrum) is represented as follows:

$S_{u_{1} u_{2}}(f)=\sqrt{S_{u_{1} u_{1}}(f) S_{u_{2} u_{2}}(f)} \gamma(\tau, f)=S_{u}(f) \gamma(\tau, f)$

where $\gamma(\tau, f)$ is the coherence function. Dragt [26], Sørensen[27] and Veers [15] suggest the following exponential form [28]:

$\gamma(\tau, f)=\exp \left(-\frac{a \cdot d(\tau) \cdot f}{\bar{U}_{h}}\right)$

In Equation 5, $a$ is the decay constant. The specific value of $a$, which is obtained by experiment, ranges from 7 20. $\bar{U}_{h}$ is the mean wind speed at the height of the hub; $d(\tau)$ is the distance between two points during time, $\tau$, as is shown in Figure 3. The calculating equation recommended by Dragt [26] is as follows:

$d(\tau)=2 r\left|\sin \frac{2 \pi f_{0} \tau}{2}\right|=2 r\left|\sin \frac{\phi}{2}\right|$

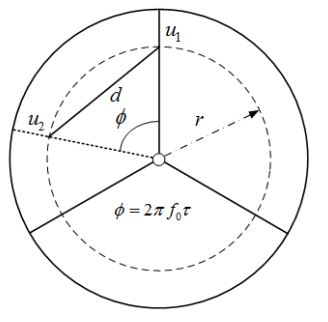

Fig.3 The geometry sketch diagram of rotational Fourier auto-spectrum

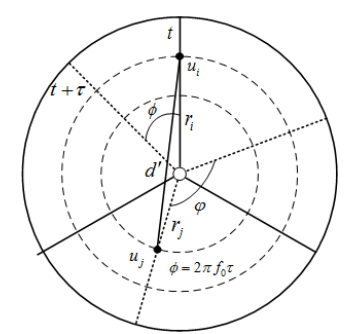

Fig.4 The geometry sketch diagram of rotational Fourier cross-spectrum 
According to Tayor frozen hypothesis [29, 30], the cross-correlation function between $u_{1}$ and $\boldsymbol{u}_{2}$ can be transformed into the auto-correlation function for any point at different times, namely, $\hat{R}_{u u}(\tau)=R_{u_{1} u_{2}}(\tau)$. Therefore, the rotational Fourier auto-spectrum at any point of blades could be obtained through Fourier transformation from auto-correlation function $\hat{R}_{u u}(\tau)$, and the specific formula is as follows:

$$
\begin{aligned}
& \hat{S}_{u u}(f)=\int_{-\infty}^{+\infty} \hat{R}_{u u}(\tau) \cdot e^{-i 2 \pi f \tau} d \tau \\
& =\int_{-\infty}^{+\infty} S_{u}\left(f^{\prime}\right) d f^{\prime} \cdot \int_{-\infty}^{+\infty} \gamma\left(\tau, f^{\prime}\right) \cdot e^{i 2 \pi\left(f^{\prime}-f\right) \tau} d \tau
\end{aligned}
$$

It can be seen in Equation 5 that the coherence function $\gamma(\tau, f)$ belongs to the triangular form of Fourier series and the period is $1 / f_{0}$. Therefore, it can be expanded into the exponential form of the Fourier series as follows:

$$
\gamma(\tau, f)=\sum_{n=-\infty}^{+\infty} t_{n}(f) \cdot e^{i 2 \pi n f_{0} \tau}
$$

In Equation $8, f_{0}$ is the rotating frequency of blades; $t_{n}(f)$ is the complex Fourier coefficient, namely, rotational mode, given in Equation 9.

$$
\begin{aligned}
& t_{n}(f)=f_{0} \int_{0}^{1 / f_{0}} \gamma(\tau, f) \cdot e^{-i \cdot 2 \pi n f_{0} \tau} d \tau \\
& =\frac{1}{2 \pi} \int_{0}^{2 \pi} \gamma(\phi, f) \cdot e^{-i \cdot n \phi} d \phi
\end{aligned}
$$

The property of Dirac delta function, namely, $\delta$ function can be represented as follows:

$\int_{-\infty}^{+\infty} e^{-i 2 \pi\left(f-f^{\prime}-n f_{0}\right) \tau} d \tau=\delta\left(f-n f_{0}-f^{\prime}\right)$

Substituting Equation 8 and Equation 10 into Equation 7, the basic equation of rotational Fourier auto-spectrum can be obtained as follows:

$$
\hat{S}_{u u}(f)=\sum_{n=-\infty}^{+\infty} t_{n}\left(f-n f_{0}\right) \cdot S_{u}\left(f-n f_{0}\right)
$$

Furthermore, Substituting Equation 9 into Equation 11, the specific formula of the rotational Fourier auto-spectrum is obtained in the following form:

$$
\hat{S}_{u u}(f)=\frac{1}{2 \pi} \sum_{n=-\infty}^{+\infty} \int_{0}^{2 \pi} \gamma\left(\phi, f-n f_{0}\right) \cdot \cos (n \phi) d \phi \cdot S_{u}\left(f-n f_{0}\right)
$$

\subsection{Rotational Fourier cross-spectrum}

In light of the above, the PNL model does not consider the correlation of fluctuating wind speed at different points on rotating blades. In addition, the SNL model considers the correlation of wind speed at different points through directly adopting the coherence function to construct the rotational power crossspectrum in the Cartesian coordinate system. It is worth noting that due to the rotational effect of blades, the method of employing the product of rotational power auto-spectrum and coherence function does not work. Herein, the correlation of the fluctuating wind speed at different points on the rotating blades is deduced in the rotating coordinate system.

It is assumed that $u_{i}$ represents the wind speed time series of any point at time, $\mathrm{t}$ and radius, $r_{i}, u_{j}$ is the wind speed time series of another point at time, $t+\tau$ and radius, $r_{j} . d^{\prime}(\tau)$ is the distant between two points, as shown in Figure 4. The equation for determining $d$ ' is given in Equation 13.

$$
d^{\prime}(\tau)=\sqrt{r_{i}^{2}+r_{j}^{2}-2 r_{i} r_{j} \cos \left(2 \pi f_{0} \tau+\varphi\right)}
$$

In this equation, $\varphi$ is the initial phase angle of the blade; when two points are on the same blade, $\varphi=0$, and the rotational Fourier cross-spectrum is described as the cross-spectrum between different points on the same blade. When two points are located on the different blades, $\varphi=2 \pi / N$, where $\mathrm{N}$ is the number of blades. For the wind turbine structures applied in this study, $\varphi=2 \pi / 3$, and the rotational Fourier cross-spectrum is expressed as the crossspectrum between different points on the different blades. Therefore, through introducing the initial phase angle $\varphi$, the rotational Fourier cross-spectrum truly considers the correlation of the fluctuating wind speed on the blades rotating plane.

The derivation of rotational Fourier cross-spectrum is similar to the rotational Fourier auto-spectrum, and only the coherence function $\gamma^{\prime}(\tau, f)$ transforms into the following form:

$$
\gamma^{\prime}(\tau, f)=\sum_{n=-\infty}^{+\infty} t_{n}^{\prime}(f) \cdot e^{i \cdot n\left(2 \pi f_{0} \tau+\varphi\right)}
$$

The corresponding complex Fourier coefficient, namely, the rotational mode can be expressed into the following equation:

$$
\begin{aligned}
& t_{n}^{\prime}(f)=f_{0} \int_{0}^{1 / f_{0}} \gamma^{\prime}(\tau, f) \cdot e^{-i \cdot n\left(2 \pi f_{0} \tau+\varphi\right)} d \tau \\
& =\frac{1}{2 \pi} \int_{0}^{2 \pi} \gamma^{\prime}(\phi, f) \cdot e^{-i \cdot n(\phi+\varphi)} d \phi
\end{aligned}
$$

Finally, the basic and detailed forms of the rotational Fourier crossspectrum are given in Equation 16 and Equation 17, respectively.

$$
\begin{aligned}
& \hat{S}_{u_{i} u_{j}}(f)=\sum_{n=-\infty}^{+\infty} t_{n}^{\prime}\left(f-n f_{0}\right) \cdot S_{u}\left(f-n f_{0}\right) \cdot e^{i \cdot n \cdot \varphi} \\
& \hat{S}_{u_{i} u_{j}}(f)=\frac{1}{2 \pi} \sum_{n=-\infty}^{+\infty} \int_{0}^{2 \pi} \gamma^{\prime}\left(\phi, f-n f_{0}\right) \cdot \cos (n \phi) d \phi \cdot S_{u}\left(f-n f_{0}\right)
\end{aligned}
$$

It can be seen from Equation 11 and Equation 16 that the rotating Fourier spectrum is superposed of infinitely classical fluctuating wind origin spectrum, obtained by translating to integer times of rotating frequency $f_{0}$, multiplied by corresponding rotational mode. Therefore, it is not accurate to apply the classic fluctuating wind speed spectrum (auto-power spectrum) such as the Von Karman spectrum and the Kaimal spectrum for predicting the extreme loads and fatigue loads.

It is worth mentioning that when the order of the Fourier series, $\mathrm{n}$ is sufficiently large, both Equation 12 and Equation 17 involve the integral of high oscillatory functions. In order to avoid the occurrence of Runge oscillation, the classical Filon method [31] is applied to solve this type of integral.

\subsection{The Comparison between Origin Spectrum and Rotational Fourier Spectrum}

In order to compare the difference between the origin spectrum and rotational Fourier spectrum, the measured wind turbine structure at Clayton in New Mexico, conducted by Connell [11], is taken as a case. In the case provided by Connell, the height of the hub is $30.5 \mathrm{~m}$. The radius of the blades is $19 \mathrm{~m}$. The mean wind speed at the height of the hub is $8.21 \mathrm{~m} / \mathrm{s}$. The rotating frequency of blades is $0.667 \mathrm{~Hz}$. The longitudinal turbulence standard deviation is $0.79 \mathrm{~m} / \mathrm{s}$. The ground roughness length is $0.005 \mathrm{~m}$. The longitudinal turbulence integral scale is $112 \mathrm{~m}$. The lateral turbulent integral scale is $45 \mathrm{~m}$. In addition, the Von Karman fluctuating wind spectrum is adopted as the origin spectrum of the rotational Fourier spectrum. The specific equation is as follows [23]:

$$
\frac{f \cdot S_{u}(f)}{\sigma_{u}^{2}}=\frac{4 f \cdot L_{u} / V_{h u b}}{\left[1+70.8\left(f L_{u} / V_{h u b}\right)^{2}\right]^{5 / 6}}
$$

In this equation, $V_{\text {hub }}$ represents the mean wind speed at the height of hub; $\sigma_{\mathrm{u}}$ represents the longitudinal turbulence standard deviation; $L_{\mathrm{u}}$ represents the longitudinal turbulence integral scale.

Based on the parameters provided above, a program was created by using MATLAB software [32]. The calculated results are shown in Figure 5 through Figure 6.

As shown in Figure 5(a), with an increase in the order $n$ (integer multiple of rotational frequency) of the origin spectrum, the spectrum peak is translated to the position which corresponds to $n$ times of rotating frequency, and each peak value is equal. Moreover, the peak values of $n$-order rotational mode are observed at the integer multiple of the rotating frequency, and with increase in order $n$, the peak values gradually decrease (see Figure $5(b)$ ). 
Compared with the Von Karman spectrum, the energy distributions of the rotational Fourier spectrum, which transfer from the low frequency range to high frequency range, produce fundamental change, as shown in Figure 6. In addition, the spectrum peak values appear at the integer multiples of the rotating frequency.

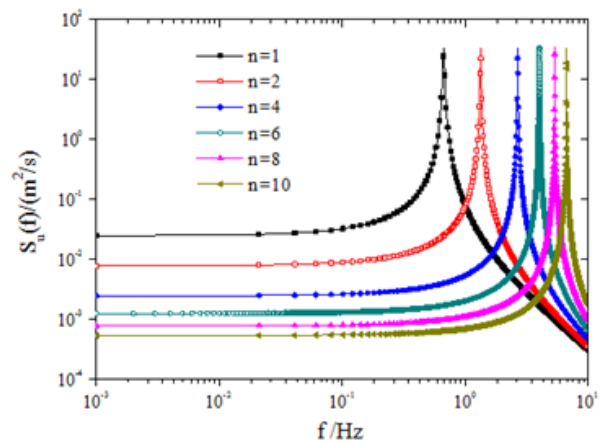

(a) n-order origin spectrum curve

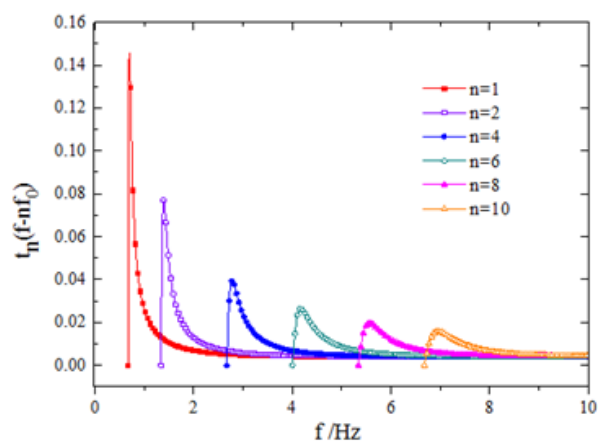

(b) n-order rotational mode curve

Fig.5 The translated origin spectrum and rotational mode curve

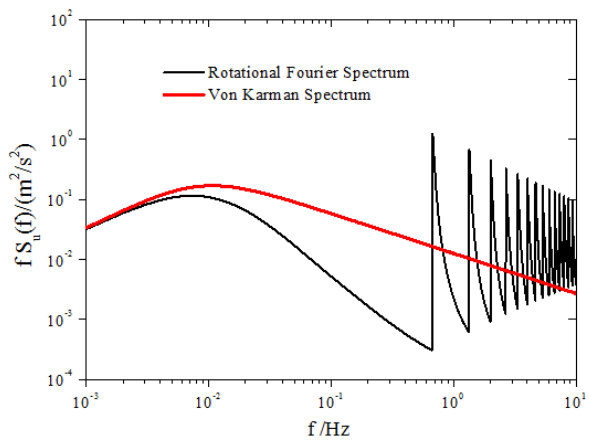

Fig.6 Comparison between Von Karman spectrum and rotational Fourier spectrum

\subsection{Optimization of Coherence Function}

It can be observed from Equation 12 and Equation 17 that the coherence function has a key influence on the rotational Fourier spectrum. Generally, the coherence function adopts Davenport exponential form. Firstly, the coherence function type in Equation 5 and Equation 6, recommended by Dragt [26], is explored. It can be observed in Figure 5(b) that the frequency of each order rotational mode only starts from the corresponding integer multiples of the rotating frequency. The reason for this is that in order to guarantee the coherence function satisfying the equation $0 \leq \gamma\left(\phi, f-n f_{0}\right) \leq 1$, the frequency must meet $f>n \cdot f_{0}$ based on the positive decay constant.

As shown in Figure 5(b), the rotational modes appear as stepped variations at the integer multiples of the rotational frequency, the case that is unreasonably held by Dragt [26]. In order to investigate the essential cause of this variation, the first-order coherence function and rotational mode varying with rotating angle $\phi$ near the rotating frequency $f_{0}$ are taken as an example, as drawn in Figure 7.

It can be seen from Figure 7 that when $f=f_{0}=0.667$, the integral of the first-order rotational mode and coherence function values in the interval $[0,2 \pi]$ are always equal to 0 and 1 , respectively. When the frequencies $f$ stay away from the rotating frequency $f_{0}$, the integral values of the first-order rotational mode, which are greater than 0 , increase first and decrease afterwards as rotating angle $\phi$ increases. In addition, the coherence function, which is obviously smaller than the corresponding values when $f=f_{0}=0.667$, decreases first and increases afterwards.

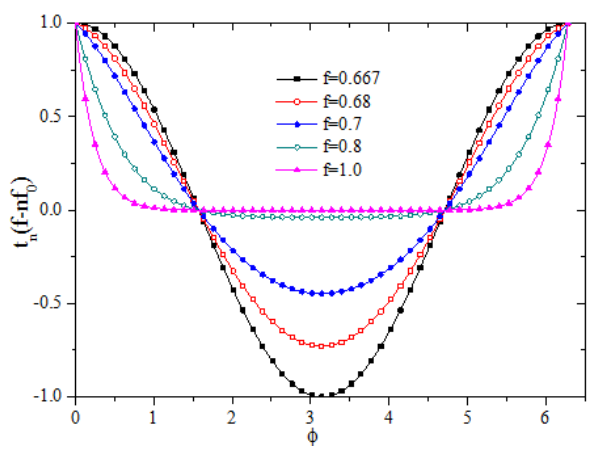

(a) Rotational mode curve

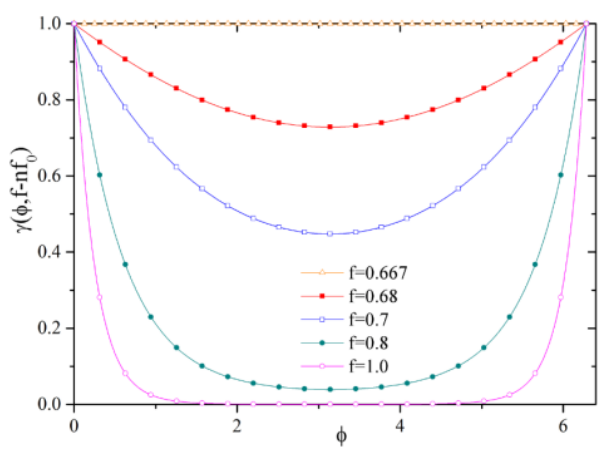

(a) Coherence function curve

Fig.7 Rotational mode and coherence function varying with rotating angle

On the whole, the reason why the rotational Fourier spectrum appear as stepped variations at the integer multiples of the rotational frequency is because the coherence function values are always equal to 1 when $f=n f_{0}$, leading to the results that each order rotational mode is always equal to 0 when $f=n f_{0}$. In fact, the $\delta$ function and constant, 1 are conjugate functions of a pair of Fourier transformations. When the frequency, $f$ is equal to $n f_{0}$, the coherence function values are always equal to 1 , hence the rotational mode function is equal to the $\delta$ function. According to the property of Dirac delta function, only when $\mathrm{n}$ is equal to 0 , this function is not equal to 0 , which contradicts the fact that each order rotational mode value is greater than 0 except when the frequency $f$ is equal to $n f_{0}$. In addition, the frequency cannot cover the whole frequency ranges. Consequently, the exponential form of coherence function proposed by Dragt [26] and Veers [15] is unreasonable, and the reasonable function form should be reconstructed.

According to the above analysis, the reconstructed coherence function should be less than 1 . Therefore, the traditional Davenport type should be modified through adding minor items. The modified coherence function suggested by IEC61400-1:1999 [33] and Burton [16] is as follows:

$$
\gamma(\phi, f)=\exp \left(-8.8 \cdot d(\phi) \cdot \sqrt{\left(\frac{f}{V_{h u b}}\right)^{2}+\left(\frac{0.12}{L_{u}}\right)^{2}}\right)
$$

Furthermore, the modified coherence function model is put forward by IEC61400-1:2005[25] and GBT 18451.1-2012 [34]. The specific formula takes the following form:

$\gamma(\phi, f)=\exp \left(-12 \cdot d(\phi) \cdot \sqrt{\left(\frac{f}{V_{h u b}}\right)^{2}+\left(\frac{0.12}{L_{u}}\right)^{2}}\right)$

where $L_{u}$ is equal to $8.1 \Lambda_{u}$ and $\Lambda_{u}$ represents the turbulent scale parameter. Here, values of $\Lambda_{u}$ at the height of hub are described as follows [25, 34]:

$\Lambda_{u}= \begin{cases}0.7 z & \mathrm{z} \leq 60 \mathrm{~m} \\ 42 m & \mathrm{z} \geq 60 \mathrm{~m}\end{cases}$

The definitions of other parameters have been described earlier. 


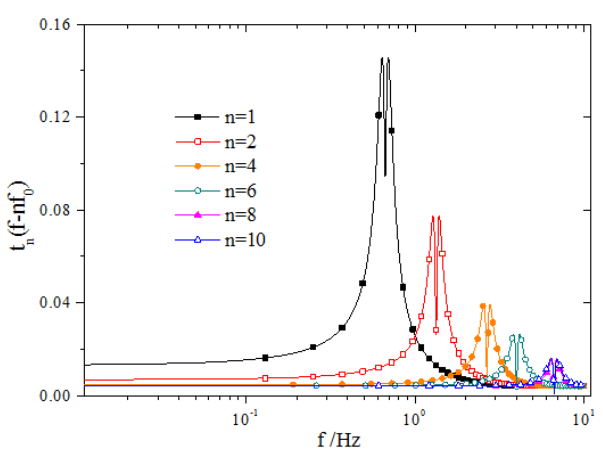

(a) Each order rotational mode when $a$ is equal to 10

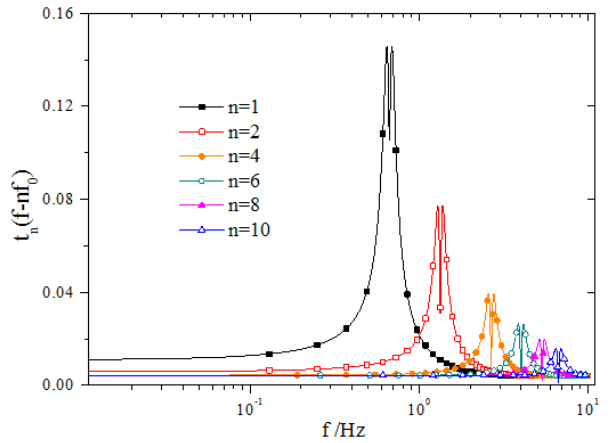

(b) Each order rotational mode when $a$ is equal to 12

Fig.8 Each order rotational mode when $a$ is equal to 10 and 12

As is shown in Equation 19 and Equation 20 and compared with coherence function form shown in Equation 6 and Equation 7, the modified formulas not only add minor terms, but also gradually adjust the decay coefficient $a$. In general, the value of $a$ ranges are from 7 to 20 . The variation laws between each order rotational mode and frequency when $a$ is equal to 10 and 12 are provided in Figure 8.

It can be shown in Fig. 8 that being different from the coherence function form shown in Equation 6 and Equation 7, each order rotational mode after adopting the modified coherence function, which distributes over the whole frequency range, are all greater than 0 when $f=n f_{0}$ even if $n=10$. Moreover, when the same decay coefficient $a$ is employed, stepped variation values induced by adopting the modified coherence function are smaller than the corresponding values caused by the traditional Davenport type of coherence function (see Figure 5(b) and Figure 8(a)). Therefore, the modified coherence function, through adding minor items is reasonable. Apart from this, it is necessary to investigate the influence of the decay coefficient on the rotational mode and rotational Fourier spectrum. Herein, based on the modified coherence function form, $a$ is selected as $7,8.8,10,12$ and 18 to study this influence, respectively.

\subsubsection{Impact on Rotational Mode and Rotational Fourier Spectrum}

The impact of the decay constant on the rotational mode function is explored first. The rotational mode values at rotating frequency $f_{0}$, corresponding to different decay constant are provided in Figure 9(a). Due to numerous data points at rotating frequency $f_{0}$, the locally enlarged drawings at rotating frequency $f_{0}$ are provided to clearly show the influence of the decay constant, as illustrated in Figure 9(b).

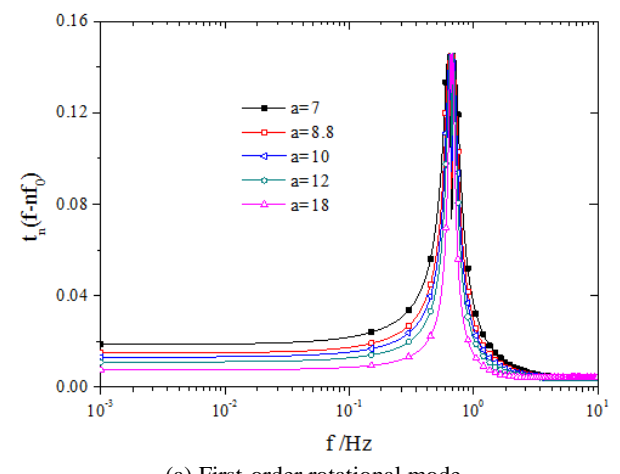

(a) First-order rotational mode

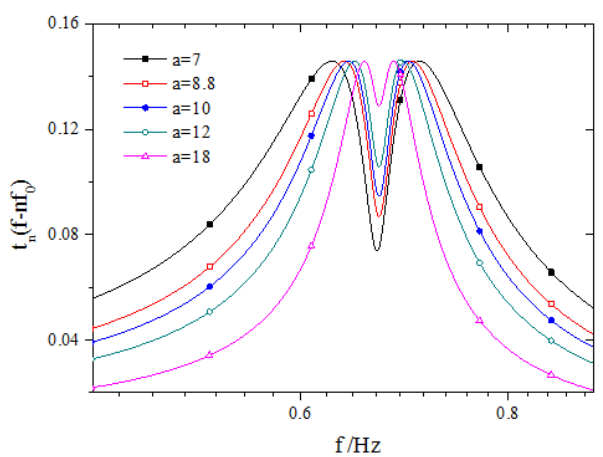

(b) locally enlarged drawings at the rotating frequency

Fig.9 First-order rotational mode corresponding to different decay

According to Figure 9, within the range staying away from $f_{0}$, the decay constant can decrease the rotational mode values. Moreover, the decay constant could lead to higher rotational mode values near $f_{0}$.It is worth noting that when the decay constant reaches the extreme value of 20, the drop-off phenomenon is least significant near $f_{0}$ (see Figure 9 and Figure 10(a)). Also, Figure 10(b) shows each order of rotational mode function when $a$ is equal to 20. It is clearly seen that compared with the decay constant adopted by IEC61400-1:2005[25] $(a=12)$, each order value of rotational mode is dramatically increased near $n f_{0}$ when $a$ is equal to 20 (see Figure 8(a) and Figure 10(b)).

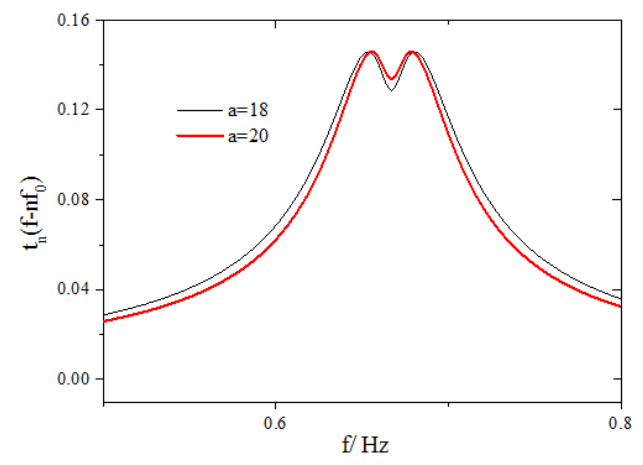

(a) First-order rotational mode when $a$ is equal to 18 and 20

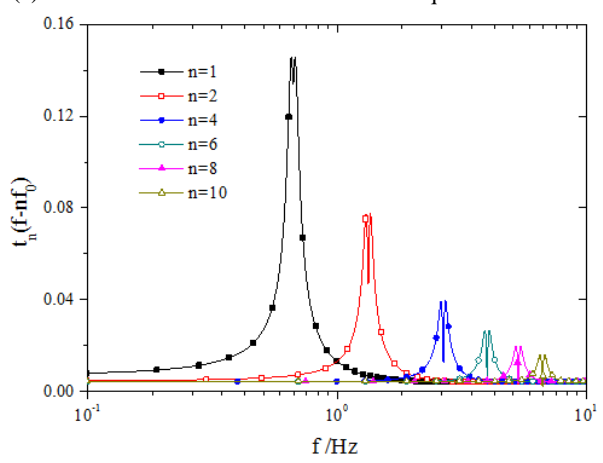

(b) Each order rotational mode when $a$ is equal to 20

Fig.10 Rotational mode values when $a$ is equal to 18 and 20

In general, the increased decay constant gives each order rotational mode the tendency to be sharp, and increases the energy at the integer times of the rotating frequency. In other words, with increase in decay constant, the drop-off phenomenon can be further eliminated.

Furthermore, the influence of the decay constant on the rotational Fourier spectrum needs to be studied, as detailed in Figure 11. Being similar to the results of rotational mode, the increased decay constant makes the energy transfer from the low frequency ranges to high frequency ranges. Based on Equation 11 and Equation 16, each rotational mode has a drop-off phenomenon at the integer times of the rotating frequency, which eventually brings about the same case for rotational Fourier spectrum. In addition, the drop-off phenomenon is least evident at the integer times of the rotating frequency when $a$ is equal to 20 


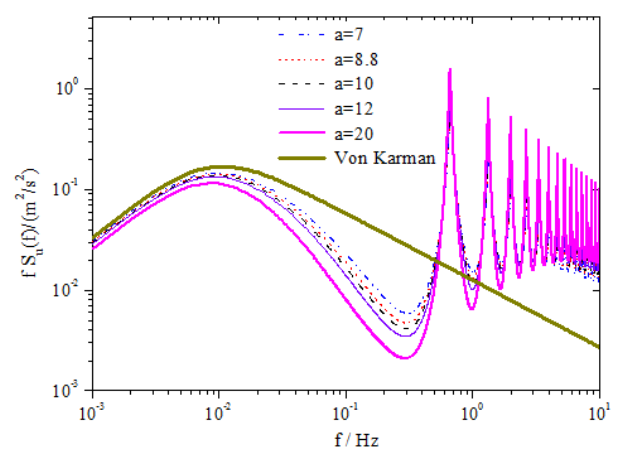

Fig.11 Rotational Fourier spectrum corresponding to different decay constant

Overall, the selection of decay constant has a great influence on the rotational mode function and rotational Fourier spectrum. The increased decay constant makes the wind field energy around blades generate redistribution, and further weakens the drop-off phenomenon. Therefore, considering that the decay constant $(a=12)$, which is applied by IEC61400-1:2005[25], still lead to significant drop-off phenomenon, the upper limit of decay constant $a=20$ is recommended and adopted in the next section.

\subsubsection{Comparison with measured data}

According to Equation 12 and the optimized coherence function, the rotational Fourier spectrum is programmed, and then the calculated results are compared to the measured rotational spectrum conducted by Connell [11]. It is worth mentioning that the measured rotational spectrum only takes the first five harmonic frequencies. This is possibly due to the fact that Nyquist frequency (half of the sampling frequency) must exceed the highest frequency of sampled signal to ensure the reconstruction of the original continuous signal. Compared results are provided in Figure 12(a). As indicated in Figure 12(a), the rotational Fourier spectrum values based on proposed model in this study and optimized decay constant have better agreement with measured rotational spectrum values.

In order to further verify the accuracy of the rotational Fourier spectrum model, this spectrum model is compared with the Connell spectrum (PNL model) as well as TurbSim calculation spectrum (based on the modified SNL model) [35], as shown in Figure 12. It can be clearly seen that the deviation values of the PNL model from the measured rotational spectrum in the wave trough are relatively larger, compared with the proposed rotational Fourier spectrum in this study. The rotational Fourier spectrum has good agreement with the TurbSim calculation spectrum, especially in the high frequency range which is important for rotational sample. This apparent difference in the low frequency range between TurbSim calculation spectrum and rotational Fourier spectrum is attributed to that the Von Karman spectrum used as origin spectrum of SNL model has lower accuracy when the frequency is smaller than the rotating frequency of blades [15]. Therefore, the rotational Fourier spectrum around the blades can accurately predict the extreme load and fatigue load of wind turbine structure.

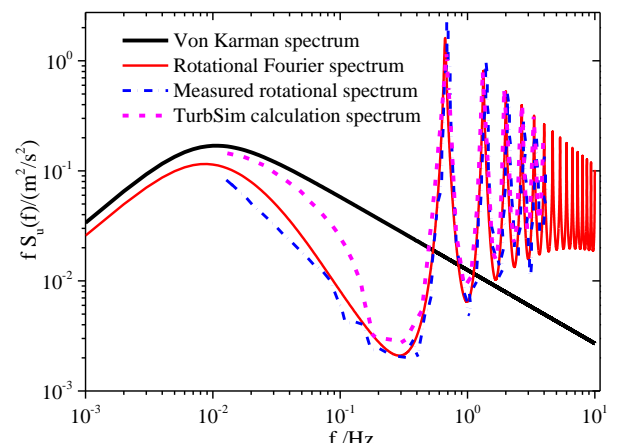

(a) Comparison with measured rotational spectrum and TurbSim calculation spectrum

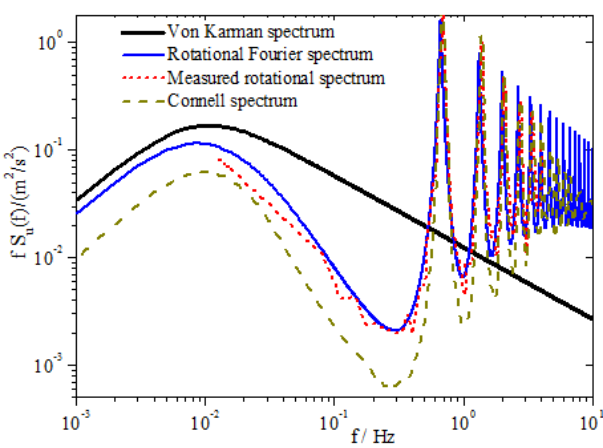

(b) Comparison with Connell spectrum (PNL model)

Fig.12 Comparison between the proposed rotational Fourier spectrum and the different rotational spectrum models

\subsection{Parameter analysis of Rotational Fourier Spectrum}

The rotational Fourier spectrum model is related to the calculated radius of the blade, rotating frequency of blades, mean wind speed at the height of hub, and the fluctuating wind speed correlations of different points. This study considers a typical pitch-controlled 1.25MW wind turbine structure, based on which parameter analyses of rotational Fourier spectrum are carried out to explore their influence laws.

In this study, it is considered that the diameter of blades is $64.35 \mathrm{~m}$; the rated rotating speed of blades is $17.8 \mathrm{r} / \mathrm{min}$; the height of the hub is $63.342 \mathrm{~m}$; the mean wind speed at the height of hub $V_{\text {hub }}$ is $12 \mathrm{~m} / \mathrm{s}$ and ground roughness length is $0.005 \mathrm{~m}$. The origin spectrum of the rotational Fourier spectrum adopts the Kaimal wind spectrum recommended by IEC61400-1:2005[25] where the detailed formula is as follows:

$$
\frac{f S_{u}(f)}{\sigma_{u}^{2}}=\frac{4 f\left(L_{u} / V_{h u b}\right)}{\left(1+6 f\left(L_{u} / V_{h u b}\right)\right)^{5 / 3}}
$$

According to the IEC standards [25], the longitudinal turbulence standard deviation $\sigma_{u}$ is $1.752 \mathrm{~m} / \mathrm{s}$ and the longitudinal turbulence integral scale, $L_{u}$ is $340.2 \mathrm{~m}$. The coherence function uses the optimized exponent form, and the specific equation is as follows:

$\gamma(\phi, f)=\exp \left(-20 \cdot d(\phi) \cdot \sqrt{\left(\frac{f}{V_{h u b}}\right)^{2}+\left(\frac{0.12}{L_{u}}\right)^{2}}\right)$

where $d(\phi)$ represents the distance between two points. For the rotational Fourier auto-spectrum, $d(\phi)=2 r \cdot\left|\sin \left(\frac{\phi}{2}\right)\right|$ and for the rotational Fourier cross-spectrum, Equation 13 is adopted.

\subsubsection{Calculated radius of blades}

As can be seen from Equation 23, the calculated radius $r$ on the blade is closely related to the coherence function, which further influences the rotational Fourier spectrum. In this study, $\mathrm{r}$ is taken as $0,10,20$ and $30 \mathrm{~m}$ to investigate this influence. The analysis results are provided in Figure 13(a).

It is clearly seen in Figure 13(a) that when the calculated radius is equal to 0 , the calculated point locates at the height of hub, and the rotating effect disappears, indicating that the rotational Fourier spectrum degenerates into the Kaimal spectrum of fixed points. Moreover, within low frequency components staying away from $n f_{0}$, the increased calculated radius can decrease the rotational Fourier spectrum values. For high frequency components near $n f_{0}$, the increased radius could lead to larger rotational Fourier spectrum values. This implies that the energy transfers from the low frequency to high frequency gradually, and high frequency oscillation becomes more and more severe. It is worth noting that when the calculated radius reaches $30 \mathrm{~m}$, the energy redistribution phenomenon is most significant. 


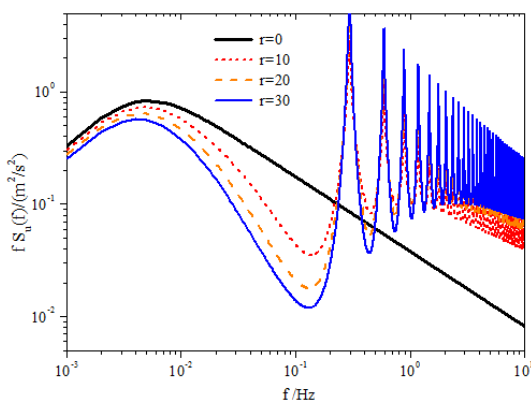

(a) The influence of calculated radius on rotational Fourier spectrum

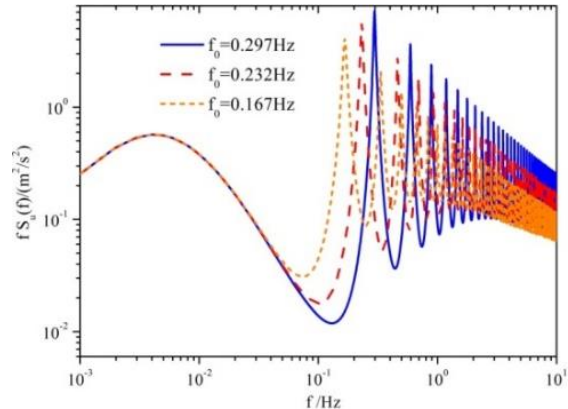

(b) The influence of rotating frequency on rotational Fourier spectrum

Fig.13 The influence of radius and blades rotating speed on rotational spectrum

\subsubsection{Rotating speed of blades}

According to Equation 12, the rotational Fourier spectrum is closely related to the rotating frequency of the blades. For the $1.25 \mathrm{MW}$ wind turbine structure studied in this study, the working speed of the rotor is $9.6 \sim 17.8 \mathrm{r} / \mathrm{min}$, and therefore the corresponding rotational frequency range is $0.16 \sim 0.30 \mathrm{~Hz}$. To study the influence of the rotating speed on the rotational Fourier spectrum, the rotating frequencies are chosen as $0.167 \mathrm{~Hz}, 0.232 \mathrm{~Hz}$ and $0.297 \mathrm{~Hz}$ and the corresponding calculated results are given in Figure 13(b).

According to Figure 13(b), the increased rotating frequency can result in larger rotational Fourier spectrum peak values, which indicates that the rotating effect of the blades becomes more significant. In addition, with an increase in the rotating frequency, the peak values of the rotational Fourier spectrum near $n f_{0}$ translate to the high frequency range side.

\subsubsection{Mean wind speed}

For the wind turbine structures in service discussed in this study, the cut-in wind speed (minimum wind speed of electrical generation) is $4 \mathrm{~m} / \mathrm{s}$, the rated wind speed (corresponding to the rated power) is $16 \mathrm{~m} / \mathrm{s}$, and the cut-out wind speed (maximum wind speed of electrical generation) is $25 \mathrm{~m} / \mathrm{s}$. To deeply understand the influence of the mean wind speed at the height of the hub on the rotational Fourier spectrum, the mean wind speed is selected as 12,18, and $24 \mathrm{~m} / \mathrm{s}$. The analysis results are shown in Figure 14(a). It is clearly seen that the increased mean wind speed increases the rotational Fourier spectrum values and leads to significant rotating effect of the blades.

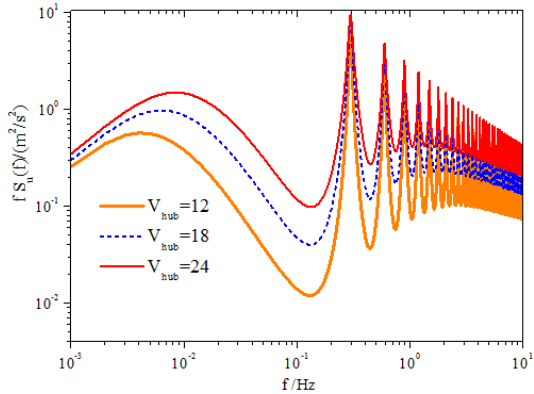

The influence of mean wind speed on rotational Fourier spectrum

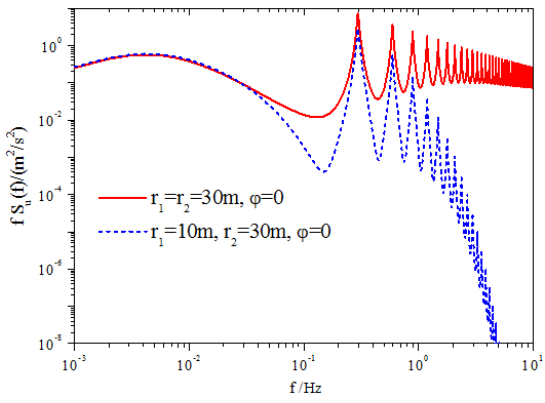

(b) The comparison of rotational Fourier spectrum for the same blade

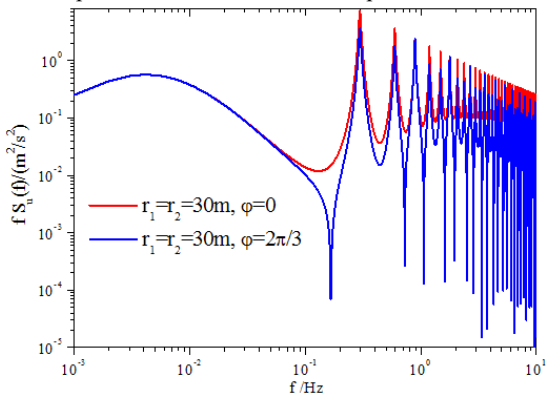

(c)

The comparison of rotational Fourier spectrum for the same radius

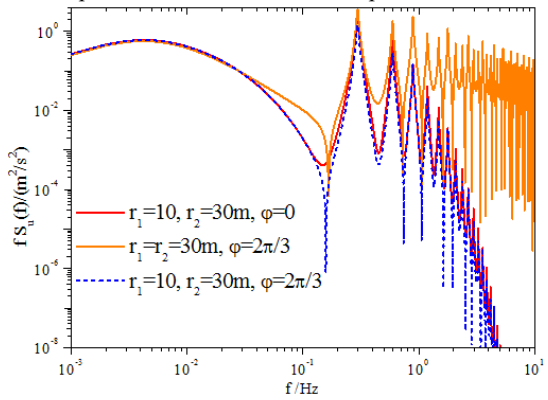

(d) The comparisons for different rotational Fourier cross-spectrums

Fig.14 The influence of mean speed and correlation between different points on rotational Fourier spectrum

\subsubsection{Correlation between different points}

The influence of the correlation between different points on the windinduced responses and fatigue issues is significant. The rotational Fourier spectrum model proposed in this study not only considers the correlation between different points located on the same blade, but also considers those located on different blades. Therefore, the rotational Fourier auto-spectrum $r_{1}=r_{2}=30 m, \varphi=0$, rotational Fourier cross-spectrum including $r_{1}=10 m, r_{2}=30 m, \varphi=0 \quad, \quad r_{1}=r_{2}=30 m, \varphi=2 \pi / 3 \quad$ and $r_{1}=10 m, r_{2}=30 m, \varphi=2 \pi / 3$ are considered to investigate the impact of the correlation. The corresponding calculated results are given in Figure 14(b) through Figure 14(d). As can be seen from Figure 14(b), for the same blade, the rotational Fourier auto-spectrum values are obviously greater than the rotational Fourier cross-spectrum values corresponding to different radii, especially within the high frequency ranges. Under the condition of the same radius, the rotational Fourier auto-spectrum values are almost the same as the rotational Fourier cross-spectrum values corresponding to different blades. However, the initial phase angle of the blade, $\varphi$ brings about a significant influence on the rotational Fourier spectrum values (see Figure 14(c)). In addition, for the rotational Fourier cross-spectrum, the amplitude of the rotational Fourier cross-spectrum corresponding to the same radius and different blades is largest, and the rotational Fourier cross-spectrum corresponding to different blades and radii leads to the smallest amplitude (see Figure 14(d)).

\section{Wind field simulation of wind turbine structure}

Owing to the strongly nonlinear property of wind turbine structures, the time-domain method is used to directly understand the dynamic characteristics and obtain more information about fatigue issues compared with the frequencydomain method. Moreover, dynamic time history analysis of wind turbine structures requires time series data of the wind speed. In general, the artificial wind field simulation method, which is economical, fast and accurate, is widely used to obtain the accurate wind speed series data.

\subsection{The application scope of turbulent wind spectrum}


be classified into two types: the fixed part such as tower and the periodic rotating parts such as the blades. As mentioned previously, the wind field around the tower is essentially different from that around the blades. For any point on the tower, wind speed is only a function of time, so its turbulent wind spectrum is the same as that of high-rise buildings. In this study, the Kaimal wind spectrum recommended in IEC61400-1:2005 [25] is adopted to simulate the turbulent wind field of the tower. For any point attached to the rotating blade, the wind speed is not only related to the time, but also the periodic variations of the spatial position coordinate. Therefore, the turbulent wind spectrum of the blades needs to consider the rotational effect. This study uses the rotational Fourier spectrum deduced in the previous section to simulate the wind field of the blades. The illustrated diagram is given in Figure 15.

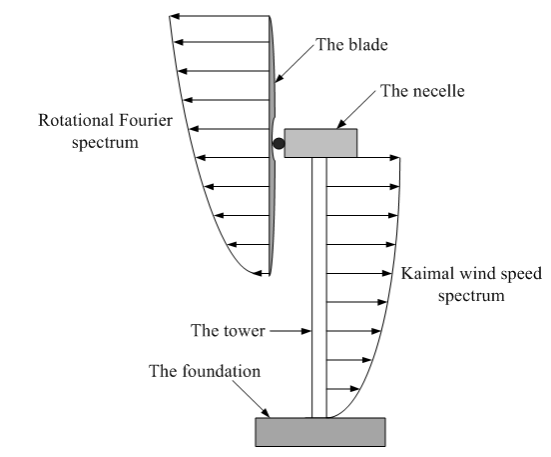

Fig. 15 Turbulent wind field model of wind turbine structures

\subsection{Wind field simulation of tubular tower}

Generally, for any point on the wind turbine structure, the wind speed consists of two parts: mean wind speed and fluctuating wind speed. The fluctuating wind field can be deemed as a four dimensional univariate random field. Furthermore, if the fluctuating wind field can be divided into the fluctuating wind field at each discrete spatial point, the fluctuating wind field can be assumed to be a multi-dimensional and multivariate stationary Gauss random process.

For mean wind speed, the exponential model describing the wind shear effect is widely used in the research field of wind turbine structures.

For wind turbine structures, the random characteristics of fluctuating wind speed at any point on the tubular tower can be described by the wind speed spectrum. As mentioned previously, the Kaimal wind spectrum is used to simulate the wind field of the tower, and shown in Equation 22. Moreover, the correlation between two points is reflected by the cross-power spectrum. Therefore, the cross-power spectrum can be described as follows:

$$
S_{u v}(f)=\sqrt{S_{u u}(f) S_{v v}(f)} \cdot \operatorname{Coh}(f)
$$

At this time, the Davenport correlation function, presented by Davenport, is used for the calculation of the correlation between different spatial points on the tower. The detailed expression is as follows [37]:

$$
\operatorname{Coh}(f)=\exp \left(-\frac{f\left[C_{y}^{2}\left(y_{1}-y_{2}\right)^{2}+C_{z}^{2}\left(z_{1}-z_{2}\right)^{2}\right]^{1 / 2}}{\frac{1}{2}\left[U\left(z_{1}\right)+U\left(z_{2}\right)\right]}\right)
$$

Where $f$ represents fluctuating wind frequency; $\mathrm{y}_{\mathrm{i}}, \mathrm{z}_{\mathrm{i}}(\mathrm{i}=1,2)$ represents the lateral and vertical coordinates of any two points on the tubular tower, and the line of two points is perpendicular to the direction of the mean wind speed; $C_{y}, C_{z}$ represents the lateral and vertical exponent decay coefficient, the values of which are recommended as 16 and 10 respectively. $U\left(\mathbf{z}_{1}\right), U\left(\mathbf{z}_{2}\right)$ represents the mean wind speed at the height of $\mathrm{z}_{1}$ and $\mathrm{z}_{2}$. In general, under the fixed fluctuating wind frequency, the correlation between two points on the tower becomes smaller with increase in distance between the two points.

\subsection{Wind field simulation of the blades}

The most significant difference between the wind field around the blades and the wind field of the tower is that the influence of the rotating effect on the wind field around the blades must be considered when the wind turbine structure is in operation. In addition, the mean wind speed for any point on the blade shows a periodic variation with the rotation of the blade.

\subsubsection{Mean wind speed}

As mentioned previously, when blades are in operation, the height of any point on the blade exhibits periodic harmonic variations. Based on the exponential model, the mean wind speed for any point at the radius $r$ of the blade can be obtained as follows:

$\bar{U}(r)=\bar{U}\left(z_{h}\right)\left(\frac{z_{h}+r \cos \phi}{z_{h}}\right)^{\alpha} \quad z=z_{h}+r \cos \phi$
In this equation, $r$ represents the radius of calculated point on the blade, i.e. the distance between the center of hub and the calculated point along the radial direction; $\phi=2 \pi f_{0} t+\varphi$ is the azimuth angle of blades at the time, $\mathrm{t}$ in the vertical rotating plane; $\varphi$ is the initial phase angle, and the location where the blade coincides with the opposite direction of gravity is defined as $0^{\circ}$ azimuth angle. It is noted that the blades rotate along the anti-clockwise direction. $z_{h}$ represents the height of hub; $\alpha$ represents the ground roughness coefficient.

\subsubsection{Fluctuating wind speed}

The random characteristics of fluctuating wind speed for any point on the blades can be reflected by the rotational Fourier spectrum model, which consists of the rotational Fourier auto-spectrum model and the rotational Fourier crossspectrum model. The rotational Fourier auto-spectrum is shown in Equation 11. Moreover, the rotational Fourier cross-spectrum model is given in Equation 16. In addition, the Kaimal wind velocity spectrum is recommended to become the origin spectrum of the rotational Fourier spectrum.

\subsection{Harmony superposition method}

The time series of the incoming wind speed for the tower and the blades are simulated through the harmony superposition method proposed by Shinozuka [38]. The time series of the fluctuating wind speed can be simulated using the following equation:

$$
u_{j}(t)=2 \sqrt{\Delta \omega} \sum_{k=1}^{j} \sum_{l=1}^{N}\left|H_{j k}\left(\omega_{k l}\right)\right| \cos \left[\omega_{k l} t-\theta_{j k}\left(\omega_{k l}\right)+\phi_{k l}\right]
$$

In this equation, $H_{j k}\left(\omega_{l}\right)$ represents elements of the lower triangular matrix $\mathbf{H}\left(\omega_{l}\right)$, which are acquired by the Cholesky decomposition of wind spectrum matrix $\mathbf{S}\left(\omega_{l}\right)$.The spectrum matrix $\mathbf{S}\left(\omega_{l}\right)$ consists of auto-power spectrum, which can be obtained using Equation 22 and Equation 11 for the tower and blades respectively, and cross-power spectrum which can be calculated using Equation 24 and Equation 16 for the tower and blades, respectively. $\omega_{k l}$ represents the double index frequency. $\phi_{k l}$ is the random phase angle. $N$ represents the sampling points. $\Delta \omega=\omega_{u p} / N$ is the frequency increment; $\omega_{u p}$ is the upper bound cutoff frequency.

\subsection{Case analysis and results}

A typical pitch-controlled $1.25 \mathrm{MW}$ wind turbine structure with three blades is taken as the case to carry out the wind field simulation. The height of the hub is $63.342 \mathrm{~m}$. The diameter of the blade is $64.35 \mathrm{~m}$. The mean wind speed at the height of hub is assumed as $12 \mathrm{~m} / \mathrm{s}$. The rotating frequency of blade is $0.297 \mathrm{~Hz}$. In order to input the aerodynamic loads acting on the wind turbine structures in the wind-induced vibration response analysis, three uniformly distributed lumped points along the radial direction of the blade are chosen as load input points and seven non-uniform distributed lumped points on the tubular tower are taken as the load input points. Therefore, the lumped points mentioned above are adopted to simulate the wind field of the wind turbine structures. The simplified dynamic analysis model of wind turbine structure is shown in Figure 16.

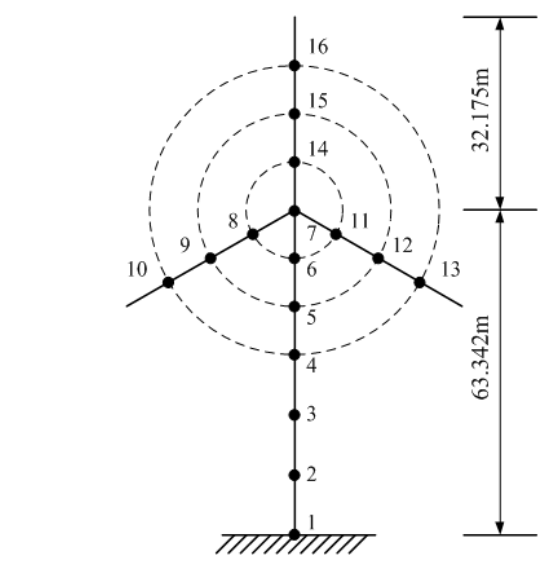

Fig. 16 Dynamic analysis model of wind turbine structure

\subsubsection{Simulated results of the tower}

As mentioned above, the mean wind speeds of the tubular tower at point 1 through point 7 are given in Table 1 . 
Table 1

The mean wind speed of the tower at each calculated point

\begin{tabular}{ccc}
\hline Number & Calculated height $(\mathrm{m})$ & Mean wind speed $(\mathrm{m} / \mathrm{s})$ \\
\hline 7 & 63.342 & 12.00 \\
6 & 55.298 & 11.71 \\
5 & 47.255 & 11.49 \\
4 & 39.211 & 11.23 \\
3 & 26.141 & 10.70 \\
2 & 13.070 & 9.85 \\
\hline
\end{tabular}

In addition, based on the FFT technology, the fluctuating wind speed time series of the tubular tower at each calculated point are obtained through the MATLAB program [32]. Based on the harmony superposition method, the upper bound cutoff frequency, $\omega_{u p}$ is set to be $8 \mathrm{~Hz}$ in the process of computing. The number of the frequency sampling points, $\mathrm{N}$ is 4096 , and the time increment, $\Delta t$ is equal to $0.0625 \mathrm{~s}$. The duration, $t$ is equal to $512 \mathrm{~s}$. The fluctuating wind speeds of the tubular tower at point 2 and point 6 (selected as a representative) are shown in Figure 17.
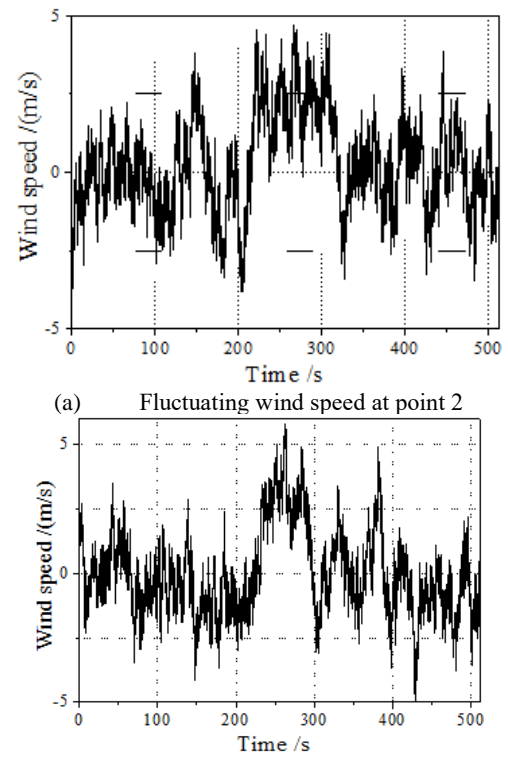

(b) Fluctuating wind speed at point 6

Fig. 17 Fluctuating wind speed of tubular tower

As indicated in Figure 17, the statistical characteristics of the simulated data are consistent with the assumptions. In order to verify the validity and reliability of the simulation method, comparative analyses between the calculated spectrum acquired through the spectrum analysis of the simulated wind speed time history, and the target wind spectrum (Equation 22) are carried out. The comparisons of wind spectrum at simulation point 2 and point 6 (selected as a representative) are shown in Figure 18

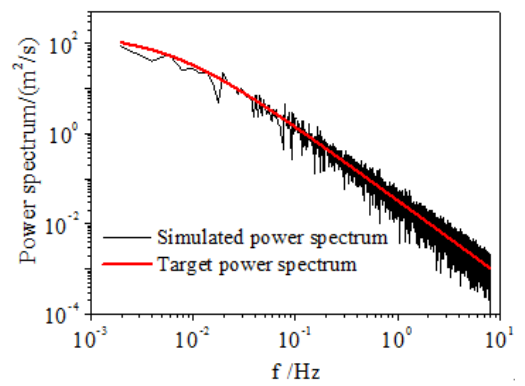

(a) The comparison diagram at point 2

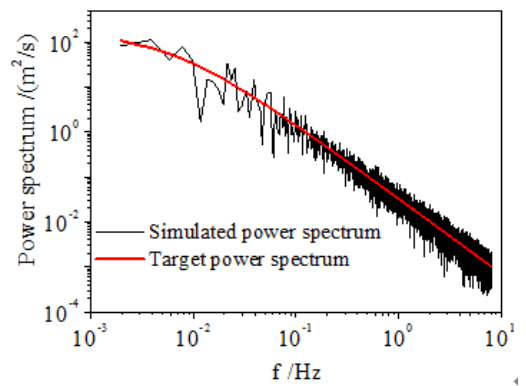

(b) The comparison diagram at point 6

Fig. 18 Comparison of simulated spectrum and target spectrum of tubular towe
It is clearly seen in Figure 18 that the spectral trend line of the simulated wind spectrum is consistent with that of the target wind spectrum, and the mean value of the spectral line has good agreement with the target wind spectrum. Therefore, the method adopted in this study is effective to simulate the fluctuating wind speed of the tubular tower. Moreover, the parameters used in this study are reasonable and practical.

\subsubsection{Simulated results of blades}

According to Equation 26 and parameters given for the case, the mean wind speed at the calculated points of blades can be determined. As the period of the mean wind speed in China is $10 \mathrm{~min}$, only the first 60 s duration are selected to clearly show the wind speed variation law against time. The reason for this is that periodic variation law of the blade mean speed is identical with those in the remaining duration. Comparisons of mean wind speed time history at different radii of the same blade are given in Figure 19(a). Comparisons of mean wind speed at the same radius of different blades are shown in Figure 19(b).

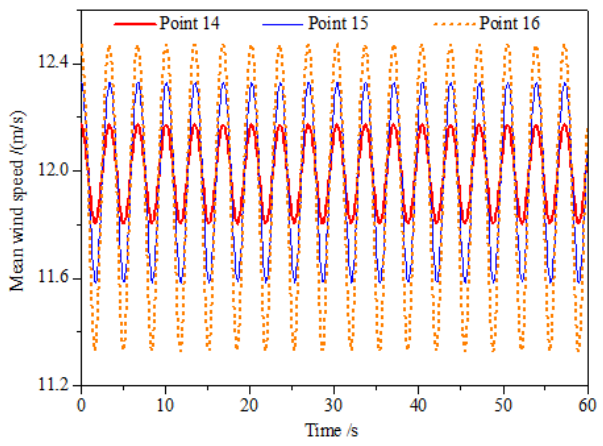

(a) The mean wind speed comparison from point 14 to point 16

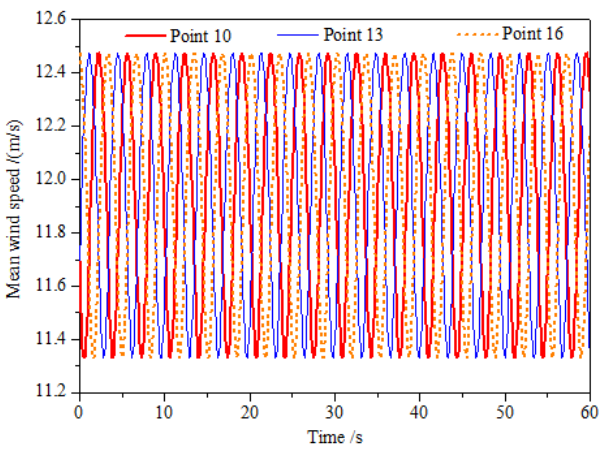

(b) The mean wind speed comparison at point 10 , point 13 and point 16

Fig. 19 The mean wind speed comparison of the blades

It can be seen from Figure 19 that being different from the mean wind speed of calculated points on the tower, the mean wind speed of the calculated points on the blades shows a periodic variation, the varying frequency of which is rotating frequency of blades. In addition, with increasing calculated radius, the mean wind speed of calculated points on the same blade gradually increase. For the calculated points on the same radius of different blades, there is a phase difference, the value of which is $2 \pi / \mathrm{N}_{\mathrm{b}}$, between the different blades. $\mathrm{N}_{\mathrm{b}}$ represents the number of blades. For the case investigated in this study, the phase difference is $2 \pi / 3$.

Based on the harmony superposition theory and FFT computing technology, the program code developed in MATLAB [32] is used to acquire the fluctuating wind speed of uniformly distributed calculated points on the blades. The simulated parameters are identical to those adopted in the tubular tower. Similarly, the fluctuating wind speed of calculated point 9,12 and 15 (selected as a representative) on the blades are provided in Figure 20.

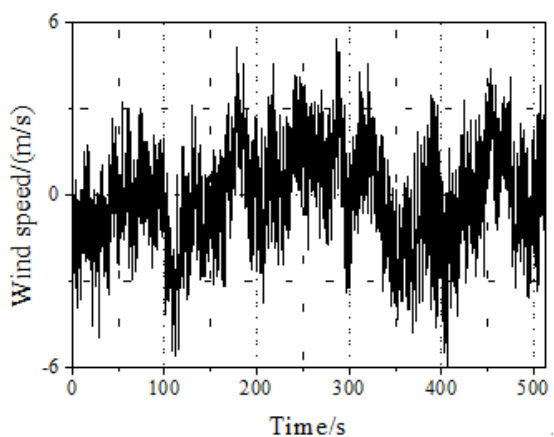

(a) Fluctuating wind speed at point 9 


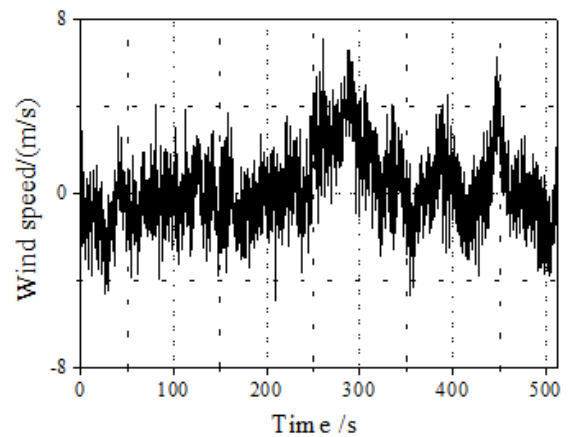

(b) Fluctuating wind speed at point 12

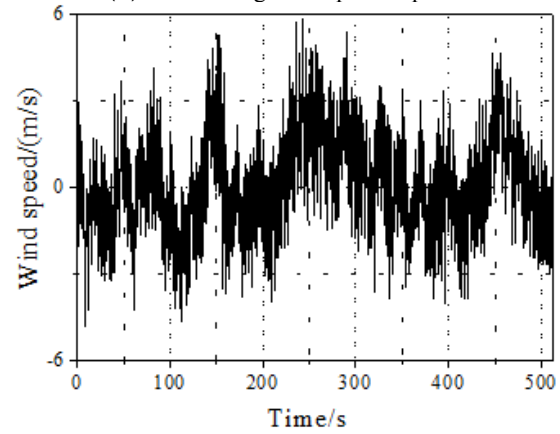

(c) Fluctuating wind speed at point 15

Fig. 20 Fluctuating wind speed of blades based on rotational Fourier spectrum

Similarly, the statistical analysis results of fluctuating wind speed are consistent with the theoretical hypothesis (see Figure 20).

Moreover, the calculations of fluctuating wind speed for corresponding points based on the Kaimal wind spectrum [3,9] are carried out, in order to consider the rotating effect of blades, as shown in Figure 21.

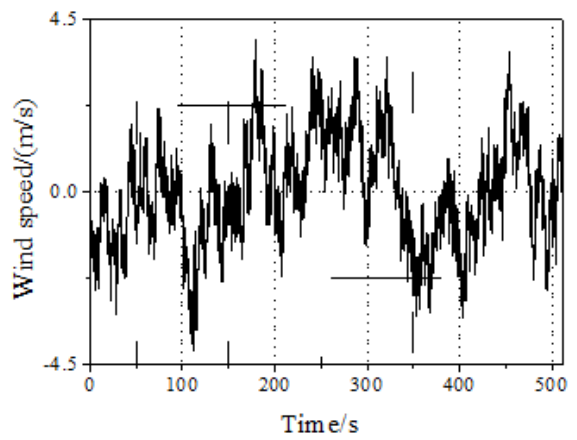

(a) Fluctuating wind speed at point 9

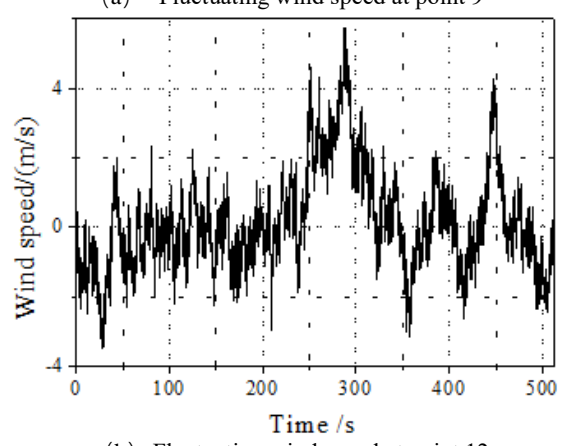

(b) Fluctuating wind speed at point 12

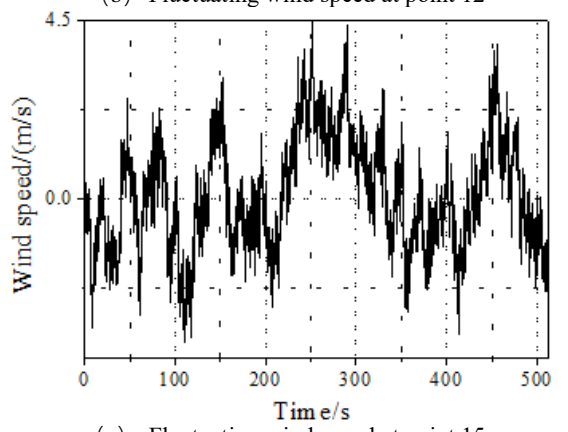

(c) Fluctuating wind speed at point 15

Fig. 21 Fluctuating wind speed of blades based on the Kaimal spectrum

As can be illustrated in Figure 21, compared with the fluctuating wind speed without considering the rotating effect of blades, the fluctuating wind speed amplitude and vibration frequency based on rotational Fourier spectrum significantly increase. The reason for this is that after considering the rotating effect of blades, the fluctuating wind speed not only reflects the fluctuating characteristics against the time, but also exhibits the spatial variation property induced by the periodic coordinate variation of calculated point on the blade. In summary, the increased amplitude and vibration frequency of fluctuating wind speed inevitably have a significant influence on the extreme load and fatigue load of the wind turbine structures. This is the essential purpose of the rotational Fourier spectrum study.

In order to confirm the effectiveness and reliability of the simulated method, the comparisons between the calculated rotational Fourier spectrum obtained by spectrum analysis of simulated fluctuating wind speed on the blades and the target rotational Fourier spectrum (Equation 12) are conducted, and the corresponding results are shown in Figure 22.

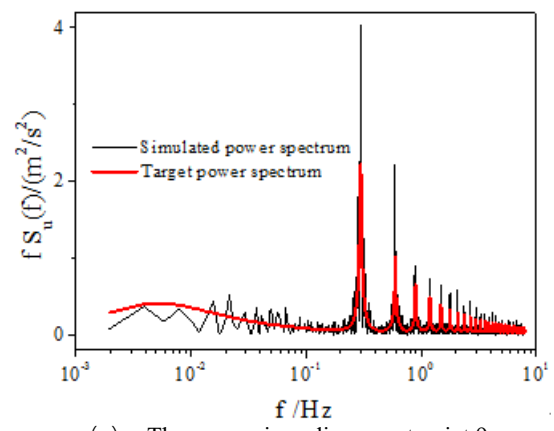

(a) The comparison diagram at point 9

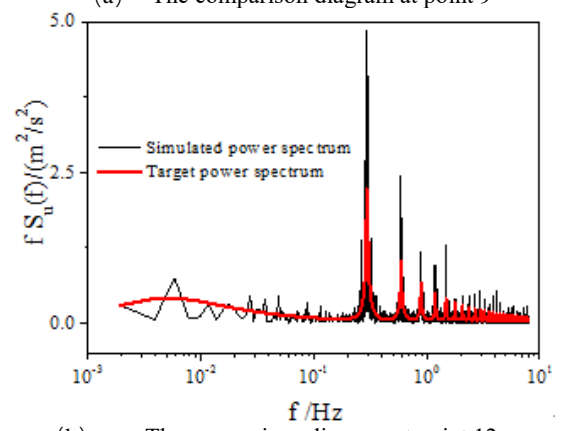

(b) The comparison diagram at point 12

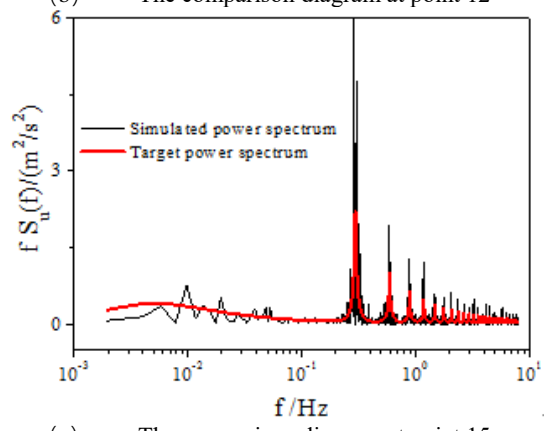

(c) The comparison diagram at point 15

Fig. 22 Comparison between the simulated spectrum and target spectrum

Comparative results from Figure 22 also show that the calculated wind spectrums give good agreement with the target rotational Fourier spectrum. In general, the fluctuating wind speed is considered as a stochastic process. As a statistical variable, the comparison between different rotational spectrums, rather than between different fluctuating wind speed time series, can be regarded more reasonable. Both Connell [11,13,39] and Veers [15] pointed out that the accuracy of their own proposed rotational spectrum model should be verified by the comparison with other rotational spectrum models or measurement data. Besides, this approach can ensure the accuracy of prediction of wind speed by guaranteeing the same statistical characteristics [13]. The rotational Fourier spectrum proposed in this study for simulating the fluctuating wind speed of blades is made comparison with the measured rotational spectrum and other important rotational spectrums, which has verified high accuracy of the proposed rotational Fourier spectrum model. From the interpretation of the findings of this study, it can be deduced that the theory and method provided in this study can accurately predict wind speed of rotating blades which can be considered as a complex phenomenon.

\section{Conclusions}

In this study, the analytical expressions of the simpler and more accurate rotational Fourier auto-spectrum and rotational Fourier cross-spectrum were systemically deduced. The optimization analysis of the coherence function and 
parameter analysis were conducted, and wind field simulation of the wind turbine structures based on the harmony superposition method were carried out. According to the analytical results, the main conclusions are noted as follows:

(1) The modified rotational Fourier spectrum model not only deals with the rotating effect of blades based on the physical mechanism, but also reflects the correlation between different points located on the same and different rotating blades. Therefore, compared with the classical wind spectrum model (origin spectrum), the rotational Fourier spectrum model presented in this study is more accurate to predict the extreme loads and fatigue loads.

(2) Compared with the Von Karman spectrum, energy distributions of the rotational Fourier spectrum are significant, especially within the high frequency ranges. Moreover, the spectrum peak values appear at the integer multiples of the rotating frequency.

(3) The optimized coherence function can not only further weaken the dropoff phenomenon of the rotational mode and the rotational Fourier spectrum, but also bring about more significant energy redistribution. In addition, compared with the PNL model, adopting the proposed rotational Fourier spectrum model and optimized coherence function can result in better agreement with the measured rotational spectrum.

(4) The increased calculating radius and rotating frequency of the blades can induce more significant energy redistribution phenomenon. Additionally, the increased mean wind speed can lead to higher spectrum values. The correlations between different points of blades are quite different.

(5) The simulations of the wind field around a tubular tower based on the Kaimal wind spectrum recommended in IEC61400-1:2005 and blades based on the rotational Fourier spectrum are reasonable and effective, by means of the harmony superposition method.

\section{Acknowledgement}

The authors wish to thank the Ministry of Science and Technology of China for financially supporting the research in the study through the Grant No. SLDRCE14-B-04.

\section{References}

[1]. Bilgili M., Yasar A. and Simsek E., "Offshore wind power development in Europe and its comparison with onshore counterpart", Renewable and Sustainable Energy Reviews, 15(2), 905-915,2011.

[2]. Heistermann C., Pavlović, M., Veljković. M., et.al. "Influence of execution tolerances for friction connections in circular and polygonal towers for wind converters", Advanced Steel Construction, 13(4), 343-360, 2017.

[3]. Ke S.T., Ge Y. J. and Wang T. G., "Wind field simulation and wind-induced responses of large wind turbine tower-blade coupled structure", The Structural Design of Tall and Special buildings, 24(8), 571-590, 2015

[4]. Huo T., Tong L.W., and Zhang Y. F., "Dynamic resoponse analysis of wind turbine tubular towers under long-period ground motions with the consideration of soil-structure interaction", Advanced Steel Construction, 14(2), 227-250, 2018.

[5]. Shan W. and Shan M., "Fatigue load estimation and reduction for wind turbine", EWEA 2015 Europe's Premier Wind Energy Event, Paris, France, 2015.

[6]. Rosenbrock H.H., "Vibration and stability problem in large turbines having hinged blades", British Electrical and Allied Industries Research Association, 1955

[7]. Connell J.R., "Turbulence spectrum observed by a fast-rotating wind-turbine blade", Report No. PNL-3426, Battelle Pacific Northwest Labs., Richland, WA (USA), 1980.

[8]. Powell D.C. and Connell J.R., "Verification of Theoretically Computed Spectra for a Point Rotating in a Vertical Plane", Solar Energy, 39(1), 53-63, 1987.

[9]. Ke S.T., Wang T.G., Ge Y.J., et.al. "Aeroelastic response of ultra large wind turbine towerblade coupled structures with SSI effect", Advances in Structural Engineering, 18(12), 20752087, 2015.

[10].Connell J.R., "The spectrum of wind speed fluctuations encountered by a rotating blade of a wind energy conversion system", Solar Energy, 29(5), 363-375,1982.

[11].Powell D.C., Connell J.R.and George, R.L., "Verification of theoretically computed spectra for a point rotating in a vertical plane", Report No. PNL-5440, Pacific Northwest Laboratory, Richland, WA (USA), 1985.

[12].Powell D.C. and Connell J.R., "A model for simulation rotational data for wind turbine applications", Report No. PNL-5857, Battelle Pacific Northwest Laboratory, Richland,WA (USA), 1986.

[13].Powell D.C. and Connell J.R., "Review of wind simulation methods for horizontal-axil wind turbine analysis", Report No. PNL-5903, Battelle Pacific Northwest Laboratory, Richland,WA (USA), 1986.

[14]. Veers P.S., "Modeling stochastic wind loads on vertical-axis turbines", Report No. SAND831909, Sandia National Laboratories, Albuquerque, New Mexico (USA), 1984.

[15].Veers P.S., "Three-dimensional wind simulation", Report No. SAND88-0152, Sandia National Laboratories, Albuquerque, New Mexico (USA), 1988.

[16].BurtonT., Sharpe D.and Jenkins, N., Wind energy handbook, 2nd section, John Wiley \& Sons, New York, 2011

[17]. Kelley N.D., "Full Vector (3-D) Inflow Simulation in Natural and Wind Farm Environments Using an Expanded Version of the SNLWIND (Veers) Turbulence Code", Report No. NREL/TP-442-5225, National Renewable Energy Laboratory, 1992.

[18].He G.L., "Rotational Fourier Spectrum of Wind Turbine Systems: A Physical Model", Advanced Materials Research, 243, 730-734, 2011.

[19].Erdem E.and Shi J., "ARMA based approaches for forecasting the tuple of wind speed and direction", Applied Energy, 88(4), 1405-1414,2011.

[20].Schueller G. I.and Shinozuka M., Stochastic methods in structural dynamics, Martinus Nijhoff Publishers, Dordrecht, 1987.

[21]. Kitagawa T. and Nomura T., "A wavelet-based method to generate artificial wind fluctuation data", Journal of wind engineering and industrial aerodynamics, 91(7), 943-964, 2003.

[22]. Kittel C., Elementary Statistical Physics, John Wiley \& Sons, New York, 1958.
[23]. Von Karman T., "Progress in the statistical theory of turbulence", Proceedings of the National Academy of Sciences of the United States of America, 34(11), 530-539, 1948

[24].Kaimal J.C.,Wyngaard J.C., Izumi Y., et al. "Spectral characteristics of surface-laye turbulence", Quarterly Journal of the Royal Meteorological Society, 98(417),563-589, 1972.

[25].IEC61400-1, Wind Turbines Part 1:Design requirements,: International Electrotechnical Commission, Geneva, 2005

[26]. Dragt J.B., "Load fluctuations and response of rotor systems in turbulent wind fields", Report No. ECN-172, Netherlands Energy Research Foundation, Petten, 1985.

[27]. Sørensen P., Hansen A.D., Rosas P.A.C., "Wind models for simulation of power fluctuations from wind farms", Journal of Wind Engineering and Industrial Aerodynamics, 90(12), 1381 1402,2002

[28].Smilden E., Sørensen A.and Eliassen L., "Wind Model for Simulation of Thrust Variations on a Wind Turbine", Energy Procedia, 94, 306-318, 2016.

[29].Taylor G.I., "The spectrum of turbulence", Proceedings of the Royal Society of London A:Mathematical, Physical and Engineering Sciences, 164(919), 476-490, 1938

[30]. Arany L., Bhattacharya S., Macdonald J., et al. "Simplified critical mudline bending moment spectra of offshore wind turbine support structures". Wind Energy, 18(12), 2171-2197, 2015.

[31].Filon L.N.G., "On a Quadrature Formula for Trigonometric Integrals", Proceedings of the Royal Society of Edinburgh, 49(1), 38-47, 1928.

[32]. Mathworks Inc., Matlab User's Guide, Natick, MA, 2016.

[33].IEC61400-1, Wind Turbine Generator Systems Part 1: Safety Requirements, International Electrotechnical Commission, Geneva, 1999.

[34].GB/T 18451.1, Wind turbine generator systems-Design requirements, Standardization Administration of the People's Republic of China, Beijing, 2012. (in Chinese).

[35].Jonkman J. and Kilcher L., Turbsim User's Guide, National Renewable Eenergy Laboratory, 2012

[36].Liu Y., Qian H.L. and Fan F., "Reflector wind load characteristics of the large all-movable antenna and its effect on reflector surface precision", Advanced Steel Construction, 13(1),1$29,2017$.

[37].Davenport A.G., "The dependence of wind load on meteoro-logical parameters", Proceedings of the International Research Seminar on Wind Effects on Building and Structures, University of Toronto, Ottawa,1967.

[38].Shinozuka M. and Jan C.M., "Digital Simulation of random processes and its applications", Journal of sound and vibration, 25(1), 111-128, 1972.

[39].Connell J.R., "Turbulence spectrum observed by a fast-rotating wind-turbine blade", Report No. PNL-4083, Battelle Pacific Northwest Labs., Richland, WA (USA), 1981. 